\title{
An extension of modified-operational-due-date priority rule incorporating job waiting times and application to assembly job shop
}

\author{
P G AWATE * and P V SARAPH ${ }^{+}$ \\ *Industrial Engineering \& Operations Research, Indian Institute of Technology, \\ Bombay 400076 , India \\ +Present address: Institute of Development Studies, University of Sussex, \\ Brighton, BN1 9RE, UK \\ e-mail: [awatepg, prasadv]@me.iitb.ernet.in
}

\begin{abstract}
The well-known priority dispatching rule MOD (Modified Operational Due Date) in job shop scheduling considers job urgency through ODD (Operational Due Date) and also incorporates SPT(Shortest Processing Time)effect in prioritising operationally late jobs; leading to robust behaviour in Mean Tardiness (MT) with respect to tightness/looseness of due dates. In the present paper, we study an extension of the MOD rule using job-waiting-time based discrimination among operationally late jobs to protect long jobs from excessive delays by incorporating an 'acceleration property' into the scheduling rule. Formally, we employ a weighted-SPT dispatching priority index of the form: (Processing time) $/$ (Waiting time) $^{\alpha}$ for operationally late jobs, while the priority index is ODD for operationally non-late jobs; and the latter class of jobs has a lower priority than the former class.

In the context of Assembly Job Shop scheduling, some existing literature includes considerable focus around the concept of 'Staging Delay', i.e., waiting of components or sub-assemblies for their counterparts for assembly. Some existing approaches attempt dynamic anticipation of staging delay problems and re-prioritisation of operations along converging branches. In the present paper, rather than depending on such a centralised and largely backward scheduling approach, we consider a partially decentralised approach, endowing jobs with a priority index yielding an 'acceleration property' based on a 'look-back' in terms of waiting time, rather than 'look-ahead'. For the particular case, in our proposed rule, when $\alpha$ is set at zero and when all jobs at a machine are operationally late, our rule agrees with MOD as both exhibit the SPT effect.

In simulation tests of our priority scheme for assembly job shops, in comparison with leading heuristics in literature, we found our rule to be particu-
\end{abstract}

\footnotetext{
* For correspondence
} 
larly effective in: (1) minimising conditional mean tardiness, (2) minimising 99-percentile-point of the tardiness distribution, through proper choice of $\alpha$. We also exploit an interesting duality between the scheduling and queueing control versions of the problem. Based on this, some exact and heuristic analysis is given to guide the choice of $\alpha$, which is also supported by numerical evidence.

Keywords. Scheduling; assembly job shops; queueing; waiting times; dynamic priorities.

\section{Introduction}

In job shop scheduling, meeting due dates is a crucial objective. Due date related priority dispatching rules essentially are based on a primarily backward scheduling approach. This can, however, lead to some uneven loading of machines over time. This cannot be avoided even if due dates are internally set because in the setting of a dynamic job shop (that is to be governed by dispatching priority rules), no sequential due date setting procedure for jobs (in the order of their arrival) can anticipate all possible conflicts among jobs for resources over time, even in a non-probabilistic situation.

Uneven loading of machines over time can be associated with uneven rates of job flows between machines and increased waiting times for jobs, due to greater variability in the arrival process for jobs coming to a machine. The well-known Modified Operation Due Date (MOD) rule proposed and studied by Baker (1984) judiciously incorporates the SPTeffect when there exist operationally late jobs at a machine. This leads towards locally maximising job flow rates past the machine, and such mobility can partially help to lessen the high ups and downs in machine loading over time in a purely backward scheduling approach based on ODD alone. Thus, although SPT is a local dispatching rule (associated with minimising mean flow time past a machine), the SPT-effect as built into the MOD rule indeed turns out to give low Mean Tardiness (MT) in job shop scheduling as such. The robustness of MOD (in respect of varying tightness of due dates) relates to the fact that ODD relates to minimising maximal operational lateness, while for operationally late jobs, minimising their mean flow time correlates with minimising their mean tardiness. Baker (1984) had also anticipated consideration of MOD rule in assembly job shops.

In connection with our observations on the impact of SPT-effect as above in a job shop, it may also be stated that a study by Russell \& Taylor (1985) has suggested that the performance of a pure SPT rule improves as the product structure (in assembly job shop) grows taller (where in fact there would exist a greater need for job mobility in the sense described above). Concern over the uneven machine loading due to purely backward scheduling strategies has also been expressed in the work of Wein \& Chevalier (1992).

On the whole, the incorporation of the SPT effect as in the MOD rule due to Baker (1984) leads to beneficial effects in job shop scheduling with due dates, and this can be interpreted or motivated in different ways as indicated above.

In assembly job shops (as opposed to pure or component job shops) there is the phenomenon of staging delay (Adam et al 1987), i.e., waiting of a component for the 


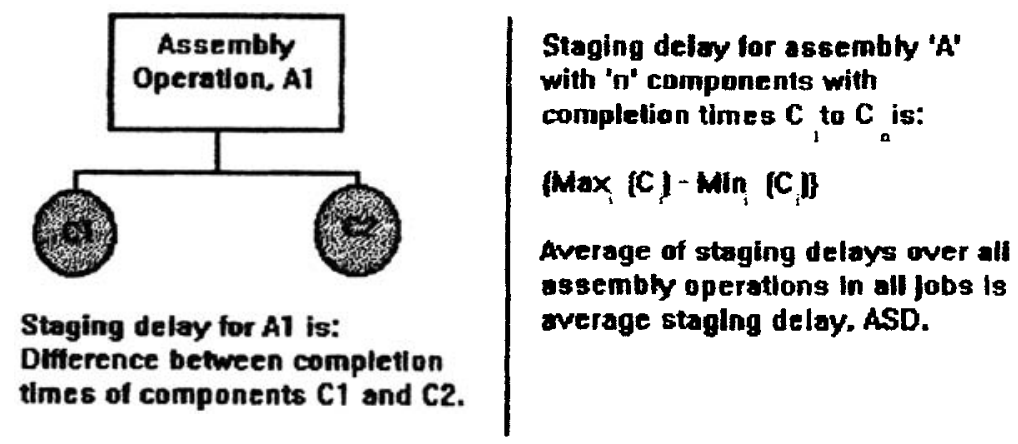

Figure 1. Staging delay in assembly jobs.

completion of other components or sub-assemblies with which it is to be assembled (figure 1).

A number of reports in the literature have attempted to devise priority dispatching rules to overcome ill effects of staging delay (Huang 1984; Adam et al 1987; Fry et al 1989). Fry et al (1991) also give a succinct summary of major findings in earlier works. A common theme in a majority of these works involves partial anticipation of staging delay problems ahead in some forms and consequent re-prioritising, using rather centralised strategies. In such schemes, delays in some branches of an assembly can lead to some loss of priorities for operations along other branches, in effect promoting a tendency for delays on those other branches with respect to the (original) ODDs. Such proliferation of possible delays can lead to undesirable consequences. There appears to be no dominant strategy emerging, robust with respect to depth/breadth of product structures as well as looseness or tightness of due dates etc.

Thus we find that on the one hand the MOD rule has not been studied in the context of assembly job shops, and on the other hand a variety of rules studied for assembly job shops in recent literature do not seem to have put forth a reliable approach to the problem while they attempt some anticipation of staging delays ahead in time. In our approach for controlling the phenomenon of staging delays, we employ a certain look-back strategy (rather than look-ahead), rooted in the fact that long operational tardiness of a job would normally be associated with a long waiting time at some machine for some operation on the job (possibly upstream). Before discussing the motivation and logic of our approach (in $\S 3$ ), we first indicate our proposed rule form in $\$ 2$.

\section{Proposed rule}

Let's define a job in general as an assembly. This is represented as a directed convergent tree whose nodes represent operations and arcs indicate immediate precedence. The root of the tree is the job's final operation. An operation having more than one immediate predecessor is an assembly operation. With each immediate predecessor, say $p$, of an assembly operation, is associated a component of that assembly. Components can be defined as maximal linear strings of operations which are mutually exclusive and such that only the first operation in the string may be an assembly operation, e.g. in figure 3 below in $\S 4$ 
the components are indicated as operation strings A, B, C and D. The notion of components is also vital to the concept of staging delay in assembly job shops, as illustrated in figure 1.

Typically, several operations on different jobs may compete for the same facility or machine at any time. An operation can qualify for competition for a machine's time only when all its predecessor operations are completed. Only when this happens, is an operation considered to be waiting for its required machine. In this context, if an operation in a job is waiting at a machine, and cannot possibly be completed within the operation's due date, then the said job is referred to as an operationally late job by the said machine.

Before defining our proposed rule, we assume that the modified operational due dates have been defined. Baker (1984) had indicated that the MOD concept, defined first for serial structures for jobs, could be usefully extended to assembly jobs. Here, we assume that job due dates have been generated based on total work content along critical path (TWKCP) as has generally been the case in literature (e.g. Adam et al 1987; Fry et al 1989 etc.). An operation's due date (ODD) is analogously generated based on the job due date and total work content along the path from the operation to job completion excluding the operation in question. Given ODDs, the MODs are defined following Baker (1984).

We propose a priority dispatching rule of the following form.

- Operationally non-late jobs to be ranked by increasing ODD (which is same as MOD here) and to form the lower priority class;

- Operationally late jobs (which are waiting since they became eligible for processing at the machine) to form the higher priority class; and jobs in this to be ranked by increasing value of the priority index:

\section{(Processing time) $/\left(\right.$ Waiting time) ${ }^{\alpha}$,}

where $\alpha$ is the single control parameter (positive). Mathematically, for operation $i$ of job $j$ we define (with reference to table $\mathrm{A} 2$ in the appendix for symbol definitions):

- $A T i, j=$ arrival (eligibility) time of operation $(i, j)$ at its required machine. Also, $\operatorname{MOD}_{i, j}$ gets defined at any time (before starting operation $i$ of $j$ ) at $T_{\text {now }}$ as the maximum of $\mathrm{ODD}_{i, j}$ and $\mathrm{EFT}_{i, j}$ (which is equal to $T_{\text {now }}+t_{i, j}$ ).

We shall refer to our rule as MODWIP-modified operational due date rule incorporating waiting time induced prioritization (figure 2). Extreme values of $\alpha$, viz. zero and infinity, impart SPT and FCFS priorities respectively to operationally late jobs. Incidentally,

Thus,

$$
\begin{aligned}
& \text { Priority Index }(\mathrm{PI})_{i, j}=\left\{\mathrm{t}_{i, j} /\left(\mathrm{T}_{\text {now }}-\mathrm{AT}_{i, j}\right)^{\alpha}\right\}, \quad \text { if, }(i, j) \text { is operationally late } \\
& =\mathrm{ODD}_{i, j} \\
& (0 \leq \alpha \leq \infty) \\
& \text { otherwise. }
\end{aligned}
$$

Figure 2. Modification of MOD using waiting induced acceleration. 
$\alpha=-\infty$ would correspond to LCFS priority; but we are not interested in $\alpha<0$. Some analysis concerning the role of $\alpha$ is given in $\S 6$ later.

This type of interpolation between SPT and FCFS does not appear to have been studied in the literature, although well known rules in literature include:

(a) Truncation of SPT by FCFS (among jobs with waiting time exceeding a limit) which forms a higher priority class;

(b) truncation of FCFS by SPT (when queue length at a machine exceeds a limit).

We sought a more elegant way of interpolating between SPT and FCFS than truncation of SPT by FCFS and vice versa. At one extreme we know that SPT minimises $F_{\text {avg }}$ and $W_{\text {avg }}$ and at the other extreme, it is easy to see the following:

Lemma 1. In static single server scheduling FCFS minimises $F_{\max }$.

[This can be seen as a corollary of the result that EDD minimises $L_{\max }$; by defining the job due dates as the job arrival times themselves.]

We have considered the FCFS rule as a limiting member of the family of the weightedSPT rules, with priority indices $\left\{p / w^{\alpha}\right\}$ as $\alpha$ goes to infinity. It turns out, as we shall see in $\S 6$, that this interpolation has interesting consequences; including the fact that as $\alpha$ goes to infinity the behaviour of the rule comes to an essential agreement with the behaviour of FCFS, in the case where waiting times are very large as compared to the processing times.

Comprehensive surveys of dispatching priority rules are given by Conway et al (1967); Gere (1966); Day \& Hottenstein (1970); Panwalkar \& Iskander (1977); Blackstone et al (1982) and Haupt (1989). For a comprehensive account of fundamental rules and algorithms in sequencing and scheduling, readers may refer to Lawler et al (1993).

Having defined our rule form, we now turn to some aspects of its motivation.

\section{Preliminary motivation for using waiting times in dynamic prioritization}

A simple motivation for our rule was as follows. Among the operationally late jobs at a machine, the ones likely to suffer large tardiness eventually, under the MOD rule, include the rather long jobs (long operation on the machine). Such long jobs would suffer a continued discrimination under SPT priority (among operationally late jobs). Our rule gives a redressal to such long and operationally late jobs, conditional upon their waiting time, so as to curtail the long tail of the tardiness distribution. The concern for the long tail here is obviously important in controlling staging delay (where any assembly operation waits for arrival of all of its components).

The use of waiting time in a dispatching priority index may be said to incorporate a simple look-back feature, which perhaps may appear to include redundant information if one were to look at the overall control decision problem in the framework of a Markovian Decision Process. Yet the fact remains that among the class of reasonably simply defined dispatching priority rules, the use of look-back information in a rule cannot be readily said to be inadmissible (in a decision theoretic sense). In fact look-back has been used 
effectively in the pioneering works due to Jackson and others (referred to in Conway et al 1967 and Kleinrock (1975)); where priority index was an affine function of waiting time. Moreover, such look-back was used precisely out of concern for "the upper tail of the waiting time distribution" (p. 180, Conway et al 1967). In our proposed rule form, we seek a weighted-SPT type priority index and use exponentiation of waiting time in our index, with a single control parameter. Concerning the choice of $\alpha$ in our rule form, we give some exact and heuristic analysis in $\S \S 6$ and 7 . For the bulk of our experimentation, we used $\alpha=0.5$; and the results of this, in comparison with several well-known priority dispatching rules are given in $\S 4$ below.

\section{Dispatching priority rules used in the experiment}

While considering dispatching priority for an operation in an assembly job, if we keep in view only the (unique) directed path from the said operation to the root node of the concerned assembly tree, then conventional job shop (non-assembly) priority dispatching rules are immediately applicable (as default options).

On the other hand, an intrinsic complication in assembly job shop scheduling exists as follows. In defining dispatching priority rules, it would be desirable to know to what extent the waiting time encountered along one path to the root node of an assembly tree has correlation with the waiting time encountered along other similar paths; while any such correlation generally cannot be independent of the priority dispatching rules used at various machines. In fact, reliable anticipation of waiting times concerns transient analysis of a (dynamic priority) queueing network, which is practically out of question. Therefore,

Table 1. Characteristics of well-known priority rules used for comparison in experimentation.

\begin{tabular}{|c|c|}
\hline Rule & Remarks \\
\hline TWK & $\begin{array}{l}\text { Consistent priority across the machines; lowers MFT \& MTD; long jobs face continual } \\
\text { discrimination, so long tail of tardiness distribution }\end{array}$ \\
\hline EDD & $\begin{array}{l}\text { Consistent priority across the machines; does not recognise operational lateness; not } \\
\text { effective with respect to MTD, if due dates are tight }\end{array}$ \\
\hline FCFS & $\begin{array}{l}\text { Discourages job passing (overtaking), thereby avoiding high variability in flow time and } \\
\text { tardiness; in a single machine static scheduling, FCFS minimises } F_{\max } \text { (exactly as EDD } \\
\text { minimises } L_{\max } \text { ) (a simple to derive, but not explicitly referred fact, which throws light } \\
\text { on the behaviour of our rule, MODWIP, when } \alpha \text { goes to infinity) }\end{array}$ \\
\hline MSLK & $\begin{array}{l}\text { Slack is difference between due date and earliest finish time; jobs with lower slack get } \\
\text { higher priority; protects long jobs from excessive delays; not effective at lowering mean } \\
\text { tardiness; has certain anti-SPT behaviour (Baker 1984) }\end{array}$ \\
\hline MOD & $\begin{array}{l}\text { Maximum of ODD and EFT for that operation; effective in minimising the conditional } \\
\text { mean tardiness due to SPT effect, when jobs are operationally late, but long tail of tardiness } \\
\text { distribution not controlled }\end{array}$ \\
\hline TWK-IR & $\begin{array}{l}\text { Refer figure } 3 \text {. Priority is based on min TWK and ties broken using max importance } \\
\text { ratio; tries to maintain parity in progress; given two components of an assembly with long } \\
\text { strings of operations, progress through operations along one branch gets de-prioritised } \\
\text { unless requisite progress occurs along the other branch (component) and vice versa; some } \\
\text { instability in this local control of staging delay can affect job tardiness. }\end{array}$ \\
\hline
\end{tabular}


even defining a simple yet sound scheme for updating operational due dates, is not an easy problem in an assembly job shop.

In view of the two reasons cited above, it is not surprising that generally in testing dispatching rules for assembly job shops, a majority of contending rules are from conventional job shop literature; as rather little seems to be known about robust dispatching rules for assembly job shops.

Given below is a list of main basic factors or statistics which are very widely used as building blocks in dispatching rules in job shops (as borne out by well-known surveys such as Panwalkar \& Iskander (1977)) and a set of rules related to each basic factor.
(a) Work content,
SPT, LPT, MWKR, LWKR.
(b) Due date,
EDD, ODD, MOD.
(c) Arrival time,
FCFS, LCFS.
(d) Operational milestones, ODD, MOD, OSL.
(e) Slack,
MSLK, OSL.
(f) Parameter ratios.
CR, COVERT.

For comparing our proposed rule, we have used certain rules from this list and also some promising rules from assembly job shop scheduling literature, e.g. Fry et al $(1989,1991)$. The rule given by Adam et al (1987) was neither considered by Fry et al (1989), nor here, as it leads to a biased account of the unfinished work along other parallel branches in a job, and more so when there are many levels of assembly. Rules considered here are described in table Al of the appendix and certain relevant observations are given in table 1.

RNOP a Remalning number of operatlons along that branch till endproduct level.

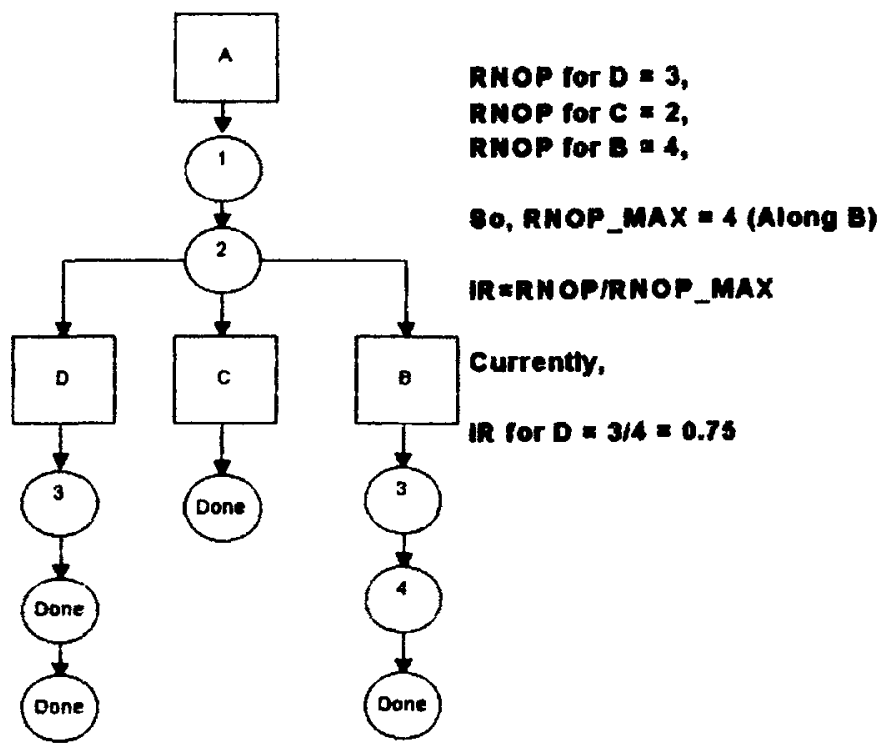

Figure 3. Concept of importance ratio (IR - refer to Fry et al 1991). 


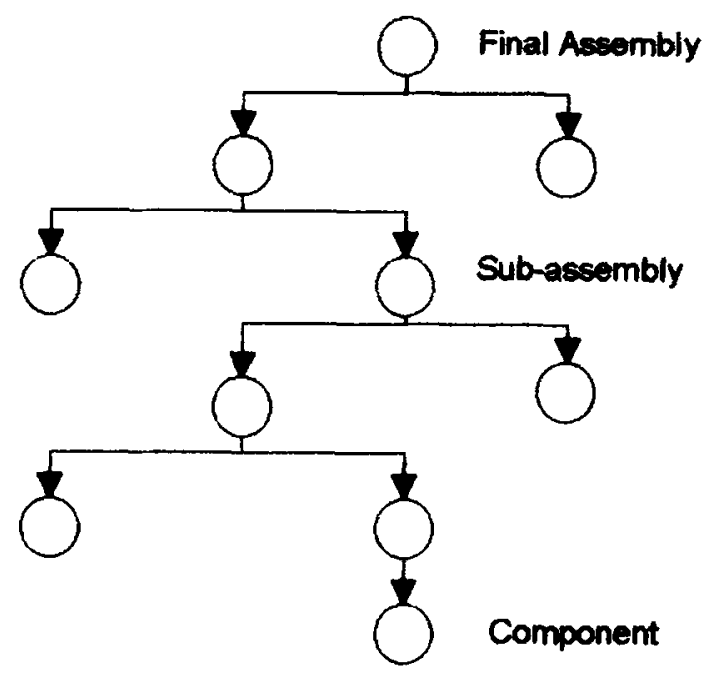

Figure 4. Tall product structure.

\section{Numerical data and results}

In our experimentation, we were interested, among other things, in the effect of product structure on the behaviour of different scheduling rules. Therefore, a common set of three product structures, representative of tall, mixed and flat structures, was used for all scheduling rules. These are illustrated in figures 4,5 and 6 . The other data on assembly jobs were generated probabilistically as is usually done in related literature. Table 2 gives the complete scheme for the generation of random jobs. Some elaboration follows the table.

We follow the assumption of Poisson job arrivals and exponential processing times as is the standard practice in literature (e.g. Baker \& Kanet 1983, Baker 1984). Poisson job arrivals imply a memoryless arrival process so that there is no question of use of, or bias due to, information on past or future arrivals (in expectation) or in choice of scheduling decisions. Choice of exponential processing time distribution is helpful here as it exhibits

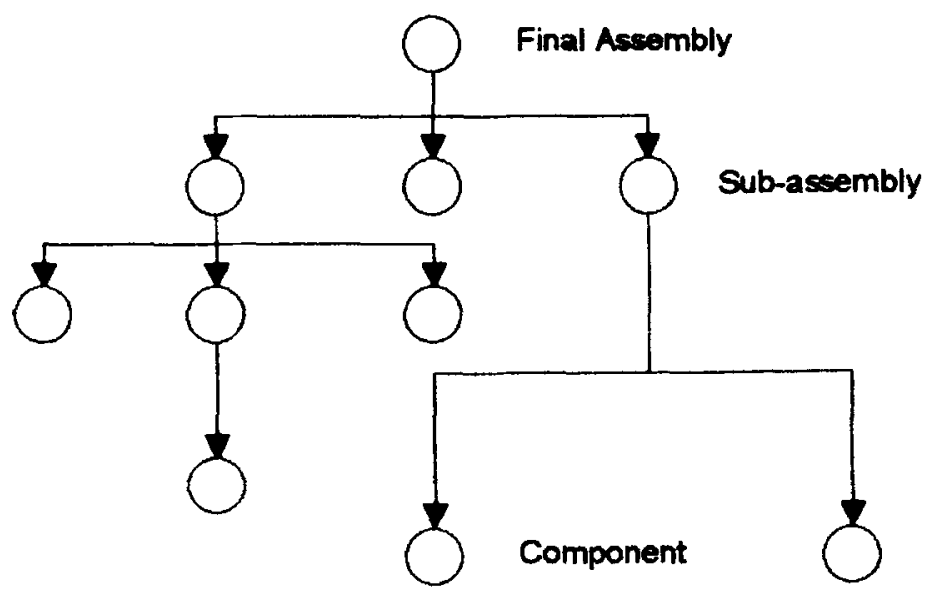

Figure 5. Mix product structure. 


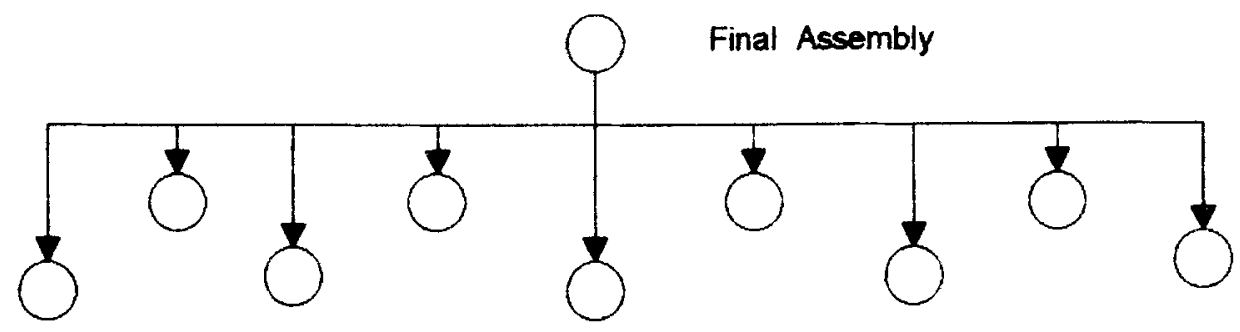

\section{Components}

Figure 6. Flat product structure

a long tail. The relative goodness of priority dispatching rules is not likely to be affected by choice of processing time distribution as long as ample variability in processing times and sufficient queueing exist in the system.

The values of mean processing time $(5 \mathrm{~h})$ and mean inter-arrival rate $(12 \mathrm{~h})$ were fixed based on an intended aggregate shop loading or utilisation around 0.6. Staging delay for components would contribute significantly to components waiting in the system, in addition to the waiting of components for a machine after they become scheduleable. It was found that system utilisation around $60 \%$ still caused $O\left(10^{2}\right)$ components waiting in the system on the whole, and this level of congestion in the system was adequate for bringing out differences among different dispatching rules without causing extremely long stabilisation periods for the system.

Tables 3, 4 and 5 give numerical results for the fourteen dispatching rules tested in respect of the six performance criteria. The details of the notations are given in tables A1 and $\mathrm{A} 2$.

Table 2. Simulation set-up.

\begin{tabular}{lcl}
\hline Parameter & Value & \multicolumn{1}{c}{ Remark } \\
\hline Number of machines & 20 & Non-identical \\
Product structures & 3 & Refer to figures 5, 6 \& 7 \\
Number of components per end product & 10 & Includes sub-assemblies \\
$\begin{array}{l}\text { Distribution for number of operations per } \\
\text { component [range] }\end{array}$ & Uniform [1,5] & \\
$\begin{array}{l}\text { Distribution for routing of a component among } \\
\text { machines [range] }\end{array}$ & Uniform [1,20] & \\
$\begin{array}{l}\text { Process of job arrivals [mean inter-arrival time] } \\
\text { Distribution for proc. time for any oprn. [mean] }\end{array}$ & Poisson [12 h] & \\
$\begin{array}{l}\text { Due date tightness factor } \\
\text { Length of each simulation run }\end{array}$ & 6 & Includes set-up times \\
Run-in period length & 12000 & DD = arvl + proc*6 \\
Interval (period) between successive instants of & 2000 & End products \\
system statistics collection & $8 \mathrm{~h}$ & End products \\
Value of $\alpha$ & &
\end{tabular}


Table 3. Results for tall product structure.

\begin{tabular}{lcrrrrr}
\hline Rule & MFT & MTD & CMT & NTJ \% & NINTN & ASD \\
\hline S/OPN & 458.86 & 9.01 & $\mathbf{7 2 . 8 6}$ & 0.123 & $\mathbf{3 3 2}$ & 158.9 \\
TWK-FIFO & 467.33 & 19.47 & $\mathbf{1 0 9 . 7 4}$ & 0.177 & $\mathbf{4 6 4}$ & 160.2 \\
TWK-IR & 476.39 & 21.82 & $\mathbf{1 1 5 . 3 7}$ & 0.189 & $\mathbf{4 9 0}$ & 154.5 \\
IR-S/OPN & 463.91 & 16.44 & $\mathbf{9 7 . 0 2}$ & 0.169 & $\mathbf{3 8 1}$ & 142.6 \\
IR-TWK & 458.09 & 15.54 & $\mathbf{9 9 . 1 6}$ & 0.156 & $\mathbf{3 8 2}$ & 140.7 \\
MS-IR & 456.84 & 17.70 & $\mathbf{1 1 1 . 0 8}$ & 0.159 & $\mathbf{4 9 1}$ & 153.0 \\
MS-TWK & 478.16 & 24.00 & $\mathbf{1 2 3 . 0 2}$ & 0.195 & $\mathbf{4 9 3}$ & 159.6 \\
TWK & 419.56 & 6.53 & $\mathbf{8 3 . 6 3}$ & 0.078 & $\mathbf{3 5 1}$ & 150.4 \\
EDD & 463.4 & 8.04 & $\mathbf{6 8 . 7 5}$ & 0.117 & $\mathbf{3 3 5}$ & 163.7 \\
FCFS & 458.14 & 6.78 & $\mathbf{7 3 . 1 5}$ & 0.092 & $\mathbf{3 2 6}$ & 155.9 \\
MSLK & 447.61 & 11.53 & $\mathbf{9 0 . 5 4}$ & 0.125 & $\mathbf{3 5 7}$ & 159.9 \\
MODWIP & $\mathbf{4 5 5 . 8 5}$ & $\mathbf{5 . 5 8}$ & $\mathbf{6 4 . 7 5}$ & $\mathbf{0 . 0 8 6}$ & $\mathbf{3 0 7}$ & $\mathbf{1 5 6 . 8}$ \\
RAN & 487.11 & 28.62 & $\mathbf{1 3 0 . 1 1}$ & 0.107 & $\mathbf{5 4 5}$ & 171.9 \\
MOD & 460.03 & $\mathbf{8 . 3 3}$ & $\mathbf{7 8 . 8 1}$ & 0.105 & $\mathbf{3 0 3}$ & 159.7 \\
\hline
\end{tabular}

\section{Regarding choice of $\alpha$}

Since our dispatching priority rule form $p / w^{\alpha}$, where $p$ is processing time and $w$ is waiting time undergone, relates to the SPT/C or weighted SPT rule (Conway et al 1967; Baker 1974), we first explore certain implications of this analogy.

Consider first an M/G/1 queue with static priority classes (Conway et al 1967; Jaiswal 1968 ; Klienrock 1975). For class ' $k$ ' jobs, assume there is a positive constant $c_{k}$ representing a weighting factor for flow times of class $k$ and $p_{k}$ is a constant processing time for jobs in class $k$. It is known that in this situation with static non-preemptive priorities among classes based on

$$
\operatorname{Min}_{k}\left(p_{k} / c_{k}\right)
$$

Table 4. Results for mixed product structure.

\begin{tabular}{lccrccc}
\hline Rule & MFT & MTD & CMT & NTJ \% & NINTN & ASD \\
\hline S/OPN & 431.50 & 24.64 & $\mathbf{7 7 . 9 2}$ & 0.316 & $\mathbf{3 7 4}$ & 144.2 \\
TWK-FIFO & 428.21 & 36.55 & $\mathbf{1 1 0 . 7 4}$ & 0.330 & $\mathbf{5 2 2}$ & 140.1 \\
TWK-IR & 445.17 & 42.81 & $\mathbf{1 1 4 . 0 4}$ & 0.375 & $\mathbf{4 8 6}$ & 139.3 \\
IR-S/OPN & 431.72 & 33.46 & $\mathbf{9 7 . 3 3}$ & 0.343 & $\mathbf{3 8 9}$ & 129.9 \\
IR-TWK & 439.69 & 33.06 & $\mathbf{9 6 . 1 3}$ & 0.344 & $\mathbf{4 1 6}$ & 129.4 \\
MS-IR & 421.59 & 32.59 & $\mathbf{1 0 2 . 5 4}$ & 0.317 & $\mathbf{4 4 2}$ & 136.3 \\
MS-TWK & 438.98 & 38.04 & $\mathbf{1 0 4 . 0 2}$ & 0.365 & $\mathbf{4 3 0}$ & 138.6 \\
TWK & 405.73 & 21.26 & $\mathbf{8 5 . 9 2}$ & 0.247 & $\mathbf{3 7 1}$ & 142.5 \\
EDD & 424.88 & 23.59 & $\mathbf{7 4 . 7 5}$ & $\mathbf{0 . 3 1 5}$ & $\mathbf{3 3 5}$ & 141.9 \\
FCFS & 422.34 & 19.04 & $\mathbf{7 1 . 3 5}$ & 0.266 & $\mathbf{2 8 9}$ & 139.1 \\
MSLK & 412.70 & 24.87 & $\mathbf{8 9 . 8 5}$ & $\mathbf{0 . 2 7 6}$ & $\mathbf{3 9 3}$ & 134.6 \\
MODWIP & $\mathbf{4 1 5 . 8 2}$ & $\mathbf{1 5 . 8 0}$ & $\mathbf{6 5 . 1 7}$ & $\mathbf{0 . 2 4 2}$ & $\mathbf{2 7 4}$ & $\mathbf{1 3 9 . 1}$ \\
RAN & 467.37 & 54.82 & $\mathbf{1 2 3 . 4 9}$ & 0.386 & $\mathbf{5 9 3}$ & $\mathbf{1 4 6 . 3}$ \\
MOD & 427.90 & 23.15 & $\mathbf{7 9 . 6 0}$ & 0.290 & $\mathbf{3 8 2}$ & $\mathbf{1 4 4 . 5}$ \\
\hline
\end{tabular}


Table 5. Results for flat product structure.

\begin{tabular}{lcccccc}
\hline Rule & MFT & MTD & CMT & NTJ \% & NINTN & ASD \\
\hline S/OPN & 421.55 & 138.83 & 166.98 & 0.831 & 607 & 274.7 \\
TWK-FIFO & 441.42 & 162.42 & 199.70 & 0.813 & 661 & 267.8 \\
TWK-IR & 414.50 & 134.80 & 168.60 & 0.799 & 564 & 254.9 \\
IR-S/OPN & 410.01 & 129.88 & 161.23 & 0.805 & 557 & 249.3 \\
IR-TWK & 406.89 & 127.79 & 161.25 & 0.792 & 538 & 249.7 \\
MS-IR & 393.68 & 118.91 & 159.76 & 0.744 & 596 & 248.5 \\
MS-TWK & 429.60 & 148.76 & 181.41 & 0.820 & 619 & 263.0 \\
TWK & 389.17 & 115.04 & 156.77 & 0.733 & 582 & 245.4 \\
EDD & 382.10 & 106.53 & 140.56 & 0.757 & 502 & 258.1 \\
FCFS & 396.88 & 115.61 & 145.33 & 0.795 & 569 & 257.8 \\
MSLK & 390.94 & 113.66 & 148.96 & 0.763 & 527 & 242.9 \\
MODWIP & 383.83 & 103.75 & 135.25 & 0.767 & 482 & 252.4 \\
RAN & 495.10 & 198.42 & 255.20 & 0.853 & 673 & 277.3 \\
MOD & 386.90 & 106.90 & 137.57 & 0.777 & 528 & 249.7 \\
\hline
\end{tabular}

and FCFS within each job class, (1) is equivalent to

$$
\operatorname{Min} E\left\{\sum_{i \in I} c_{i}\right\},
$$

where the sum extends over jobs $i$ waiting in the system (at a random point in time). Applying Little's law we also know that (2) is equivalent to

$$
\operatorname{Min}\left\{\sum_{k} \lambda_{k} \cdot w_{k} \cdot c_{k}\right\},
$$

where $w_{k}$ denotes mean waiting time (in queue) for jobs in class $k$, since the average number of jobs in the machine is invariant as there is no avoidable machine idling.

Interpreting the sum in (3) as the time rate of accumulation of system cost defined appropriately, it is seen that (3) is also equivalent to

$$
\operatorname{Min} E\left\{w_{R} \cdot c_{R}\right\},
$$

where, $R$ denotes the class index of a random job coming to the system and $W_{R}$ is a random sample of a waiting time within the jobs of class $R$.

Now, let's consider our rule form

$$
\operatorname{Min}\left\{p / w^{\alpha}\right\} .
$$

Here, essentially the priority 'class' of a job changes over time while the job waits, as it depends on both $p$ as well as $w$ (which increases). Therefore strictly speaking it is not obvious whether, analogous to (4), we would have

$$
\operatorname{Min} E\left\{W_{R} \cdot\left(W_{R}\right)^{\alpha}\right\},
$$

if we use dispatching rule as in (5) and denote $W_{R}$ a random sample of waiting times of jobs among class $R$, where the class $R$ itself is drawn at random with probability 
$\lambda_{k} /\left(\Sigma_{j} \lambda_{j}\right)$ of $R$ being $k$. Writing the said random sample $W_{R}$ simply as $W$, let us write (6) as,

$$
\operatorname{Min} E\left(W^{(1+\alpha)}\right) \text {. }
$$

Conjecture. In an $\mathrm{M} / \mathrm{G} / 1$ queueing system with heavy congestion so that with overwhelming probability,

$$
W \gg p,
$$

the dynamic dispatching rule in (5) would nearly (asymptotically, in terms of ratio of objectives) achieve the objective in (7).

The reasons why we have made a statement only in the form of a conjecture are as follows.

Reason 1. The equivalence of (5) and (7) is not expected to hold if asymptotic condition of (8) does not hold. This is explained using proposition 1 below.

\section{PROPOSITION 1}

Consider a static situation where jobs $j=1,2, \ldots, n$ have already undergone waiting times $w_{j}$ respectively. Suppose the objective is to

$$
\operatorname{Min} \sum_{j}\left(W_{j}^{(1+\alpha)}\right),
$$

where, $W_{j}$ denotes the total waiting time of job ' $j$ ' just before it gets served. If, $p_{j}$ denotes processing time of job ' $j$ ', then priority rule in (5) asymptotically achieves objective in (9), if, $W_{j} \gg p_{j}$ for all $j$.

Proof of proposition 1. The proof follows simply from the usual pair-wise interchange perturbation applied to a sequence of jobs. e.g. job 1 would immediately precede job 2 in an optimal permutation only if:

$$
\begin{gathered}
p_{2} \cdot \mathrm{d} / \mathrm{d} x\left\{\left(W_{1}+x\right)^{(1+\alpha)}\right\}_{\mathrm{at} x=0} \geq p_{1} \cdot \mathrm{d} / \mathrm{d} x\left\{\left(W_{2}+x\right)^{(1+\alpha)}\right\}_{\mathrm{at} x=0}, \\
\text { i.e. } p_{2} \cdot W_{1}^{\alpha} \geq p_{1} \cdot W_{2}^{\alpha}, \\
\text { i.e. } p_{1} / W_{1}^{\alpha} \leq p_{2} / W_{2}^{\alpha},
\end{gathered}
$$

which yields the proof.

Note that in the absence of the asymptotic condition, essentially, (8) or (10) would not hold and nor would (11). That is why the objective in (7) cannot be expected to strictly follow from the rule form of (5).

Reason 2. The linking behaviour of total differences in value of potential function for Markov chains, with costs in more than one dimension and with non-homogeneous increments in different regions of the state space, is usually not amenable to sample space methods nor to the renewal theorem (which underlies both time-limiting results concerning convergence to stationary distribution for Markov chains, and for a one-dimensional 
renewal process, the limiting behaviour of local differences in the renewal function defined on the linear state-space). In fact there exist well-known open conjectures concerning asymptotic behaviour in queueing systems (e.g. the conjecture of Jackson in Conway et al 1967 , p. 181) for which the standard limit theorems normally invoked in the context of Markov chains or Markov renewal processes do not seem to suffice. In fact the only known (to us) proof of optimality of the SPT/C rule in a truly dynamic scheme of priorities (not among classes of jobs but among individual jobs present in any given system state) is due to Kakalik \& Little (1969) which exploits in great depth the duality between LP and MDP, and no comparable studies for more general objective function seem to be known.

It may be mentioned here that as $\alpha$ goes to $\infty$, what proposition 1 tells us, in the case where $p \ll W$, stands in essential agreement with the fact seen earlier that FCFS minimises $F_{\max }$. This is because in that case minimising $W_{\max }$ is practicaliy as good as minimising $F_{\text {max }}$ from the user's point of view.

Note. In the study of dynamic dispatching rules and their optimisation at least in an approximate sense, the use of Little's law is found to shed some light on the nature of optimisation as seen from a queueing control theoretic point of view. This has also been seen, in the context of another type of problem, by Awate \& Neeraj Kumar (1992).

\section{Empirical guideline regarding choice of $\alpha$}

During early stages of our experimentation, and before our analysis given in $\S 6$, we had considered $\alpha$ as powers of $2[0.5,1,2,4,8,16]$. We found values of $\alpha$ greater than one to be not promising enough to warrant further consideration. Here we suggest a heuristic explanation for this.

From a practical point of view, we seek reasonably low mean flow time, mean tardiness, conditional mean tardiness and a small value for (say) 99 percentile point of tardiness distribution (to indicate avoidance of a long tail). Also, rather than seeking to control the 99 percentile point, it could suffice to 'keep in control' the mean and variability of tardiness. Although variance of tardiness is not directly captured by any (non-central) moment of tardiness distribution, keeping in control the first two (first and second) moments of tardiness would be a reasonable objective. Now applying the heuristic arguments concerning our conjectured relation between rule in (5) and effect in (7), we are led to the choices of $(1+\alpha)$ as 1 and 2; i.e. $\alpha=0$ and $\alpha=1$.

In light of the facts and analysis concerning our objectives, we are led to a guideline for range of $\alpha$ between 0 and 1 .

Table 6. Results for tall product structure - Variation in $\alpha$.

\begin{tabular}{lcrcccc}
\hline$\alpha$ & MFT & MTD & CMT & NTJ \% & NINTN & ASD \\
\hline 0.00 & 468.46 & 10.29 & 76.89 & 0.133 & 390 & 166.8 \\
0.50 & 455.85 & 5.58 & 64.75 & 0.086 & 307 & 156.8 \\
1.00 & 468.20 & 10.06 & 81.68 & 0.123 & 394 & 164.9 \\
\hline
\end{tabular}


Table 7. Results for mixed product structure - Variation in $\alpha$.

\begin{tabular}{lcccccc}
\hline$\alpha$ & MFT & MTD & CMT & NTJ \% & NINTN & ASD \\
\hline 0.00 & 413.13 & 19.84 & 75.56 & 0.248 & 291 & 142.1 \\
0.50 & 415.82 & 15.80 & 65.17 & 0.242 & 274 & 139.1 \\
1.00 & 424.11 & 21.66 & 71.56 & 0.268 & 291 & 139.0 \\
\hline
\end{tabular}

Our current experimentation tests values of $\alpha$ as $0,0.5$ and 1 . Among these, $\alpha=0.5$ performed better. We did not seek a fine tuning of value of $\alpha$ because we expect, based on the analysis above and numerical results, that the value of $\alpha$ recommended should be a simple or robust one. As noted by Baker (1974), the SPT effect itself often has unexpected side benefits, such as considerably reducing the mean of lateness, so that the concern for $\alpha=0$ case remains strong. On the other hand choosing $\alpha=1$ corresponds to a quadratic cost of waiting in (7). Thus, $\alpha=0.5$ seems a simple and robust compromise. Results for different values of $\alpha$ are given in tables 6,7 and 8 .

\section{Conclusions}

Use of job waiting times at a machine in the dispatching priority index for operationally late jobs has been found to provide improvement in terms of tardiness measures: mean tardiness, conditional mean tardiness and 99 percentile point ${ }^{1}$ of tardiness distribution. In case of flat product structure, we got more significant improvement in 99 percentile point of tardiness distribution; here the fraction of jobs tardy was high (more than $75 \%$ )*. Here, jobs in the upper tail of tardiness distribution were in relatively large numbers, and could be expected to be among operationally late jobs and consequently influenced by our rule's discrimination based on waiting times. On the other hand, in case of tall product structures, the fraction of tardy jobs was small (of the order of $10 \%$ ). Correspondingly here, our rule, based on discrimination among operationally late jobs, got invoked less often. The improvement we got was relatively more significant in mean tardiness indicating that some operationally late jobs, probably upstream, were accelerated by our rule and thereby prevented from being tardy at the end. In case of mixed product structure, we got significant improvement in mean tardiness, conditional mean tardiness as well as 99 percentile point of tardiness distribution.

Table 8. Results for flat product structure - Variation in $\alpha$.

\begin{tabular}{lcccccc}
\hline$\alpha$ & MFT & MTD & CMT & NTJ \% & NINTN & ASD \\
\hline 0.00 & 390.73 & 112.67 & 146.10 & 0.799 & 560 & 256.3 \\
0.50 & 383.83 & 103.75 & 135.25 & 0.767 & 482 & 252.4 \\
1.00 & 392.68 & 109.34 & 141.50 & 0.785 & 544 & 255.1 \\
\hline
\end{tabular}

\footnotetext{
${ }^{1}$ For the allowance setting mechanism, namely, six times the processing time does not provide for extra allowance in case of flat structure which is exposed to higher staging delay; which leads to higher fraction of tardy jobs as compared to tall structure. This is in agreement with the general concern in the literature that staging delay is a very major factor affecting tardiness.
} 
As we had indicated earlier in the paper and in conformity with some of the earlier works cited from the literature, the SPT effect seems to play a crucial role in tall product structure and indeed we found the MOD rule to be a very strong competitor, especially in tall product structure. Our current results indicate the MOD rule giving a slightly better value for the 99 percentile point of the tardiness distribution (but not for MTD and CMT). In our further experimentation, involving variation of more number of factors, we intend to take a closer look at the cited phenomenon in tall product structures.

Regarding choice of parameter ' $\alpha$ ' in the experimentation of waiting time in our priority index, our results indicate that $\alpha=0.5$ is better than $\alpha=0$ (equivalent to SPT among operationally late jobs) and $\alpha=1$ (equivalent to FCFS among operationally late jobs). Based on certain analytical relations between queueing control problems and priority dispatching problems, we have indicated earlier the desirability of $\alpha$ lying between 0 and 1; reffecting concern for the mean and the second moment of tardiness. Based on our current findings, $\alpha=0.5$ is consistently better at tardiness measures as seen in tables 6,7 and 8 . Further study of the role of value of $\alpha$ will be part of future research.

Our new proposed rule, developed in the context of assembly job shop scheduling, gives an effective and efficient extension of the well-known MOD rule defined in the literature for job shop scheduling with due dates. On the other hand, we have derived a practically very interesting interpolation between SPT and FCFS dispatching rules and delineated its relation to a rich analogy with a queueing control problem. This also seems to warrant further study from the point of view of job shop scheduling in general.

Our findings are also essentially in agreement with some previous ones in literature that the most complicated rules in job shop scheduling are hardly the most effective ones and simple-looking rules based on sound analysis do have scope for furthering improvements.

We thank two anonymous referees for their comments and thoughtful observations which helped to improve the clarity at certain points in our paper. One of the referees has also pointed out to us a very fine paper "Efficient scheduling policies to reduce mean and variance of cycle time in semiconductor manufacturing plants" by Lu, Ramaswamy and Kumar in IEEE Trans. Semiconductor Manufacturing (1994, p. 374). While this paper shares the concern for variance of cycle times as we do, the problem environments and the approaches in their paper and ours are really quite different and therefore we have not formally included this in our actual reference list. 


\section{Appendix A.}

Table A1. Rules used in simulation.

\begin{tabular}{|c|c|c|c|c|}
\hline \multirow[b]{2}{*}{ Name } & \multicolumn{2}{|c|}{ Explanation } & \multicolumn{2}{|c|}{ Formula } \\
\hline & Primary rule & Tie-breaker & Primary rule & Tie-breaker \\
\hline S/OPN & $\begin{array}{l}\text { Slack per } \\
\text { operation }\end{array}$ & - & $\begin{array}{l}\operatorname{Min}_{i}\left[\left(d_{i} \cdot \mathrm{EFT}_{i}\right) /\right. \\
\left.\operatorname{RNOP}_{i}\right]\end{array}$ & - \\
\hline TWK-FIFO & $\begin{array}{l}\text { Total work } \\
\text { content }\end{array}$ & $\begin{array}{l}\text { First in, } \\
\text { first out }\end{array}$ & $\begin{array}{l}\operatorname{Max}\left[\Sigma t_{i}\right]: i=\text { current } \\
\text { oprn }{ }^{*} \text { to end oprn }\end{array}$ & $\operatorname{Min}_{i}\left[r_{i}\right]$ \\
\hline TWK-IR & $\begin{array}{l}\text { Total work } \\
\text { content }\end{array}$ & $\begin{array}{l}\text { Importance } \\
\text { ratio }\end{array}$ & $\begin{array}{l}\text { Max }\left[\Sigma t_{i}\right]: i=\text { current } \\
\text { oprn to end oprn }\end{array}$ & Refer to figure 3 \\
\hline IR-S/OPN & $\begin{array}{l}\text { Importance } \\
\text { ratio }\end{array}$ & $\begin{array}{l}\text { Slack per } \\
\text { operation }\end{array}$ & Refer to figure 3 & $\begin{array}{l}\operatorname{Min}_{i}\left[\left(d_{i} \cdot c_{i}\right) /\right. \\
\left.\operatorname{RNOP}_{i}\right]\end{array}$ \\
\hline IR-TWK & $\begin{array}{l}\text { Importance } \\
\text { ratio }\end{array}$ & $\begin{array}{l}\text { Total work } \\
\text { content }\end{array}$ & Refer to figure 3 & $\begin{array}{l}\operatorname{Max}\left[\Sigma t_{i}\right]: i=\text { current } \\
\text { oprn to root oprn }\end{array}$ \\
\hline MS-IR & $\begin{array}{l}\text { Modified } \\
\text { slack }\end{array}$ & $\begin{array}{l}\text { Total work } \\
\text { content }\end{array}$ & $\begin{array}{l}\text { Max. IR, if SL }<0 \\
\text { Min. SL, o/w }\end{array}$ & $\begin{array}{l}\operatorname{Max}\left[\Sigma t_{i}\right]: i=\text { current } \\
\text { opm to root oprn }\end{array}$ \\
\hline MS-TWK & $\begin{array}{l}\text { Modified } \\
\text { slack }\end{array}$ & $\begin{array}{l}\text { Importance } \\
\text { ratio }\end{array}$ & $\begin{array}{l}\text { Min. TWK, if SL >0 } \\
\text { Min. SL, o/w }\end{array}$ & Refer to figure 3 \\
\hline TWK & $\begin{array}{l}\text { Total work } \\
\text { content }\end{array}$ & - & $\operatorname{Min}_{i}\left(t_{i}\right)$ & - \\
\hline EDD & $\begin{array}{l}\text { Earliest } \\
\text { due date }\end{array}$ & - & $\operatorname{Min}_{i}\left(d_{i}\right)$ & - \\
\hline FCFS & $\begin{array}{l}\text { First come } \\
\text { first served }\end{array}$ & - & $\operatorname{Min}_{i}\left(r_{i}\right)$ & - \\
\hline MSLK & Min. slack & - & $\operatorname{Min}_{i}\left(d_{i} \cdot \mathrm{EFT}_{i}\right)$ & - \\
\hline MODWIP & Refer to $\S 2$ & - & Refer to figure 2 & - \\
\hline RAN & Random & - & $U(0,100)$ & - \\
\hline MOD & $\begin{array}{l}\text { Modified } \\
\text { operational } \\
\text { due date }\end{array}$ & - & $\operatorname{Max}\left[O D D,\left(T_{\text {now }}+t_{i, j}\right)\right]$ & - \\
\hline
\end{tabular}

* Oprn - Operation.

Table A2. Symbols and explanations.

\begin{tabular}{llll}
\hline Symbol & \multicolumn{1}{c}{ Explanation } & Formula \\
\hline$i$ & Operation & - \\
$j$ & Job & 6 \\
$k$ & Flow allowance factor & - \\
$n$ & Number of jobs (end products) & 0.5 \\
$\alpha$ & Control parameter for MODWIP & $\Sigma t_{i, j}$ over all $i$ for each $j$ \\
$t_{j}$ & Total processing time of job $j$ & $r_{j}+k^{*} t_{j}$ \\
$d_{j}$ & Due date of job $j$ & - \\
$r_{j}$ & Arrival time of job $j$ & - \\
$\mathrm{EFT}_{j}$ & Earliest finish time of job $j$ & - \\
$c_{j}$ & Completion time of job $j$ & - \\
$t_{i, j}$ & Processing time of oprn $i$ of job $j$ & - \\
$T_{\text {now }}$ & Current time & - \\
$\mathrm{RNOP}_{j}$ & Remaining number of oprns of job $j$ & \\
\hline
\end{tabular}


Table A2. (Continued)

\begin{tabular}{lll}
\hline Symbol & \multicolumn{1}{c}{ Explanation } & \multicolumn{1}{c}{ Formula } \\
\hline $\mathrm{ODD}_{i, j}$ & Operational due date of oprn $i$ of job $j$ & $\mathrm{ODD}_{i-1, j}+\left[t_{i, j} / t_{j}\right]^{*}\left(d_{j}-r_{j}\right)$ where, \\
& & $\mathrm{ODD}_{0, j}=r_{j}$ \\
$\mathrm{AT}_{i, j}$ & Arrival time of oprn $i$ of job $j$ & - \\
$\mathrm{SL}_{i, j}$ & Modified slack for oprn $i$ of job $j$ & $d_{j}-T_{\text {now }}-\Sigma t_{i, j}$ (over operations \\
& & following $i, j)$ \\
MFT & Mean flow time & $\left\{\Sigma\left(c_{j}-r_{j}\right)\right\} / n$ \\
$T_{j}$ & Tardiness of job $j$ & $\max ($ lateness, 0$)$ for job $j$ \\
MTD & Mean tardiness & $\left\{\Sigma\left(T_{j}\right)\right\} / n$ \\
CMT & Conditional mean tardiness & $\left\{\Sigma\left(T_{j}\right)\right\} / n_{t}$ \\
NTJ & Percentage of number of tardy jobs & $n_{t} / n\left[n_{t}:\right.$ all jobs such that $\left.c_{j}>d_{j}\right]$ \\
NINTN & 99\% point of tardiness distribution & Refer to figure A1 \\
ASD & Average staging delay & Refer to figure 1 \\
\hline
\end{tabular}

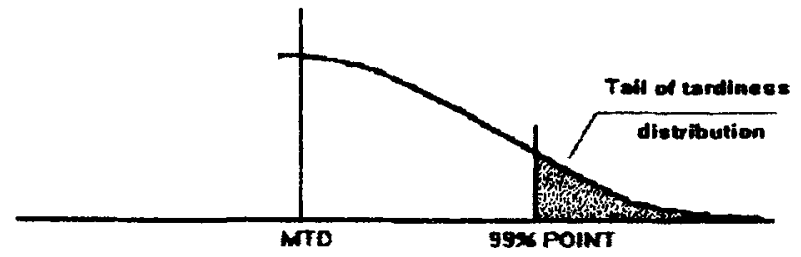

Figure A1. 99\% Point of tardiness distribution statistics.

\section{References}

Adam N R, Bertrand J W, Surkis J 1987 Heuristics for assembly job shop scheduling. IIE Trans. 19: $317-328$

Awate P G, Neeraj Kumar 1992 Development of adaptive control strategies for dispatching rules in dedicated FMS with random arrivals. Paper presented at ORSI Annual Convention at the Indian Inst. Manage., Ahmedabad

Baker K R 1974 Introduction to sequencing and scheduling (New York: John Wiley \& Sons)

Baker K R 1984 Sequencing rules and due date assignments in a job shop. Manage. Sci. 30: 1093-1104

Baker K R, Kanet J J 1983 Job shop scheduling with modified due date. J. Oper. Manage. 4: $11-22$

Blackstone J H, Phillips D T, Hogg G L 1982 A state-of-the-art survey of dispatching rules for manufacturing job shop operations. Int. J. Prod. Res. 20: 27-45

Conway R W, Maxwell W L, Miller L W 1967 Theory of scheduling (Reading, MA: AddisonWesley)

Day J E, Hottenstein M P 1970 Review of sequencing research. Nav. Res. Logist. Q. 17: 11-39

Fry T D, Philipoom P R, Russell R S 1983 Due date assignment in a multi-stage job shop. IIE Trans. 21: 153-161

Fry T D, Philipoom P R, Russell R S 1989 Dispatching rules in multi-stage assembly shops. Int. J. Prod. Res. 27: 671-686

Fry T D, Philipoom P R, Russell R S 1991 A preliminary investigation of multi-attribute based sequencing rules for assembly shops. Int. J. Prod. Res. 29: 739-753

Gere W S 1966 Heuristics in job shop scheduling. Manage. Sci. 13: 167-190

Haupt R 1989 A survey of priority rule-based scheduling. Oper. Res. Spektrum 11: 3-16 
Huang P Y 1984 A comparative study of dispatching rules in a hybrid assembly/job shop. Int. J. Prod. Res. 22: 375-387

Jaiswal N K 1968 Priority queues (New York: Academic Press)

Kakalik J S, Little J D C 1969 Dynamic operating policies for a single server facility. Technical Report No. 47, Operations Research Centre, Massachusetts Institute of Technology, Cambridge, MA

Kleinrock L 1975 Queuing systems. Vol II - Computer applications (New York: John Wiley \& Sons)

Lawler E L, Lenstra J K, Rinnooy Kan A H G, Schmoys D B 1993 Sequencing and scheduling: Algorithms and complexity. In Logistics of production and inventory: Handbooks in operations research and management science (eds) S C Graves et al (Amsterdam: North-Holland) vol. 4, ch. 9

Panwalkar S S, Iskander W 1977 A survey of scheduling rules. Oper. Res. 25: 45-61

Russell R S, Taylor B W 1985 An evaluation of sequencing rules for an assembly shop. Decision Sci. 16: 196-212

Wein L M, Chevalier P B 1992 A broader view of the job-shop scheduling problem. Manage. Sci. 38: $1018-1033$ 